\title{
Epigenetic lifestyle of Epstein-Barr virus
}

\author{
Alexander Buschle ${ }^{1}$ (D) $\cdot$ Wolfgang Hammerschmidt ${ }^{1}$ (D)
}

Received: 24 January 2020 / Accepted: 14 February 2020 / Published online: 30 March 2020

(C) The Author(s) 2020

\begin{abstract}
Epstein-Barr virus (EBV) is a model of herpesvirus latency and epigenetic changes. The virus preferentially infects human Blymphocytes (and also other cell types) but does not turn them straight into virus factories. Instead, it establishes a strictly latent infection in them and concomitantly induces the activation and proliferation of infected B cells. How the virus establishes latency in its target cells is only partially understood, but its latent state has been studied intensively by many. During latency, several copies of the viral genome are maintained as minichromosomes in the nucleus. In latently infected cells, most viral genes are epigenetically repressed by cellular chromatin constituents and DNA methylation, but certain EBV genes are spared and remain expressed to support the latent state of the virus in its host cell. Latency is not a dead end, but the virus can escape from this state and reactivate. Reactivation is a coordinated process that requires the removal of repressive chromatin components and a gain in accessibility for viral and cellular factors and machines to support the entire transcriptional program of EBV's ensuing lytic phase. We have a detailed picture of the initiating events of EBV's lytic phase, which are orchestrated by a single viral protein BZLF1. Its induced expression can lead to the expression of all lytic viral proteins, but initially it fosters the non-licensed amplification of viral DNA that is incorporated into preformed capsids. In the virions, the viral DNA is free of histones and lacks methylated cytosine residues which are lost during lytic DNA amplification. This review provides an overview of EBV's dynamic epigenetic changes, which are an integral part of its ingenious lifestyle in human host cells.
\end{abstract}

Keywords Herpesvirus $\cdot$ Chromatin $\cdot$ Infection $\cdot$ Latency $\cdot$ Reactivation

\section{Introduction}

Epstein-Barr virus (EBV) is a human herpesvirus with a DNA genome of about $165 \mathrm{kbps}$ [11]. Many known strategies of EBV mimic cellular processes and principles as the virus has coevolved with its human host. EBV has copied central mechanisms of the cell, e.g., receptor signaling, DNA replication, and gene transcription, for its own success. Thus, the viral model is a window to the cell, rich in biology and an excellent source to study epigenetic principles of key importance in metazoan cells.

This article is a contribution to the special issue on Infection-induced epigenetic changes and the pathogenesis of diseases - Guest Editor: Nicole Fischer

Wolfgang Hammerschmidt

hammerschmidt@helmholtz-muenchen.de

1 Research Unit Gene Vectors, Helmholtz Zentrum München, German Research Center for Environmental Health and German Centre for Infection Research (DZIF), Partner site Munich, Marchioninistr. 25, D-81377 Munich, Germany
EBV is also a human tumor virus [5]. It is associated with and contributes to several human tumor entities. Among them are different types of B cell lymphoma (Hodgkin's disease, Burkitt's lymphoma, non-Hodgkin's lymphomas), cancers (nasopharyngeal cancer, gastric cancers), and other malignancies. More than $90 \%$ of the world population is infected with EBV for a lifetime, whereas tumor incidence is relatively low, i.e., in the order of 200,000 annually [20,97]. Certain cofactors contribute to tumor formation, but they are mostly unknown. In all cases, EBV establishes a latent infection in the tumor cells, which express different sets of viral genes, termed viral latency programs.

In vitro, EBV preferentially infects nonproliferating, quiescent human B-lymphocytes, activates them, induces their indefinite proliferation in vitro, and establishes a strictly latent infection in them. In viral particles, the large EBV DNA is epigenetically naïve, i.e., lacks associated histones and is free of methylated $\mathrm{CpG}$ dinucleotides. Upon infection, the viral DNA is delivered to EBV's target cells and maintained as extrachromosomal plasmid copies in the nucleus. Here, the viral DNA acquires nucleosomes including histones with mostly repressive 
marks and finally a very high degree of $\mathrm{CpG}$ methylation. When and how viral DNA acquires cellular chromatin and its individual components and which cellular factors drive this process are largely unknown.

In the so-called pre-latent phase of viral infection [53] during the first 8 days (Fig. 1) when EBV reprograms the resting B-lymphocytes into activated and proliferating B blasts [74], viral transcription is pervasive, which leads to the expression of many viral genes. In contrast, in established latently infected cells, a clearly defined minimal set of very few viral genes is active. It is very likely that the deposition of cellular chromatin onto viral DNA is an essential step in controlling the program of viral transcription of the pre-latent phase ensuring the survival and continuous proliferation of EBV-infected cells long term. As a result, so-called lymphoblastoid cell lines (LCLs) emerge in which EBV establishes a stable latent infection.

Latent infection is a paradigm of all herpesviruses, but EBV excels at latency, its preferred lifestyle. In healthy EBV-positive individuals, B-lymphocytes, in particular a minute fraction of long-lived memory B cells (one in $10^{4}$ to $10^{6}$ cells), form the viral reservoir of latent infection. Viral latency has been studied in memory B cells ex vivo, tumor cells obtained from biopsies, and established tumor cell lines as well as in LCLs. LCLs and established tumor cell lines are a rich source to study all aspects of viral latency and have contributed much to understand the molecular mechanisms of viral latency. Certain cell lines such as the Burkitt's lymphoma cell lines, Akata, Raji, and P3HR1 [48, 85, 103], have also been instrumental to study EBV's escape from latency ([3, 31, 69, 86, 91]; for a selection of original works) and to identify the viral switch gene BZLF1 $[21,102]$ that can turn latently EBVinfected cells into virus factories that release viral progeny.

The key to EBV's success in infecting and persisting in its host lies in its ingenious tripartite epigenetic life cycle (Fig. 1) $[45,117]$. We have learned that the virus cannot induce virus de novo synthesis upon cellular infection but enters its so-called pre-latent phase. The virus reprograms the infected B cells, activates them to grow in size, and drives them into several rounds of intense proliferation until proliferation decelerates to a doubling time of about $30 \mathrm{~h}$. In these cells, which are now in the latent phase, no virus is synthesized, but a small subset of viral genes is expressed, and several copies of the viral genome are maintained as extrachromosomal plasmids. Upon induction of EBV's third and lytic phase, the full set of about 80 viral genes is expressed. The viral genomic DNA replicates autonomously, and viral progeny is synthesized. Infectious viral particles are released and spread horizontally to other permissive cells or are transmitted to other individuals.

Epigenetic principles govern the different expression patterns linked to the three phases of EBV's life cycle (Fig. 1). The virus has evolved to take advantage of the host cell's epigenetic machinery to first establish a stable latent infection and then to use a smart principle to escape from it.

\section{EBV's virion DNA is epigenetically naïve}

In viral particles, the large EBV DNA is epigenetically naïve, i.e., it lacks associated histones [54] and is free of methylated CpG dinucleotides ([60], Supplementary Fig. S3 in ref. [57]). This is a surprising finding but fully in line with other herpes viruses [39]. We will see later how EBV achieves this state, which is reminiscent of epigenetic events in sperm DNA during fertilization.

Sperm DNA is mostly free of nucleosomes, and compared with somatic cell DNA, the levels of histone retention are 1 and $10 \%$ in mouse and man, respectively [12]. Shortly after fertilization and prior to DNA replication or transcription in the fertilized egg, the histone variant $\mathrm{H} 3.3$ is incorporated by the histone chaperone HIRA. This step precedes the genomewide demethylation that takes place in the male pronucleus. Prior to DNA replication, $\mathrm{H} 3$ and $\mathrm{H} 4$ histones in paternal DNA, which are newly acquired from the oocyte, are acetylated, and $\mathrm{H} 3$ histones gain mono-methyl marks at K4, K9, and K27. Only after the first round of DNA replication, paternal DNA acquires tri-methylation of some of them, notably $\mathrm{H} 3 \mathrm{~K} 4 \mathrm{me} 3$, and H3K27me3 [92].

Why could this information be important for the first steps in EBV infection? It seems plausible that certain aspects are recapitulated by EBV upon infection of primary B-lymphocytes. For example, the incoming EBV DNA rapidly acquires nucleosomes in the infected cell prior to its first round of DNA replication and cell division. The molecular mechanisms are unknown, but the viral model offers an attractive, perhaps even unique, opportunity to investigate de novo chromatin assembly and regulation in a tractable system and in mammalian cells. Parallels in viral DNA in the initial phase of infection and in sperm DNA in zygotes with regard to de novo establishment of chromatin suggest that both fundamental processes might be mechanistically and biologically similar.

\section{Acquisition of cellular chromatin components upon infection}

Little is known how EBV DNA acquires chromatin constituents upon de novo infection. Presumably, the earliest action is the recruitment of histones supported by histone chaperones such as HIRA, DAXX, CAF1, ASF1, DEK, and several others, which can manage transport, location and deposition, mobilization, and replacement of nucleosomes. To our knowledge, close to nothing is known about the timing and dynamics of this step during EBV infection. We only know from preliminary experiments that the landscape of nucleosomes 
on EBV DNA is completed in B-lymphocytes within $48 \mathrm{~h}$ post infection (Mrozek-Gorska et al., unpublished). This observation suggests that nucleosome assembly is replication independent and occurs in activated cells during G1 phase and long before the onset of EBV-induced cellular DNA replication [74]. Based on this assumption, it is likely that replication-independent histone chaperones are predominantly involved in this early step of chromatinization.

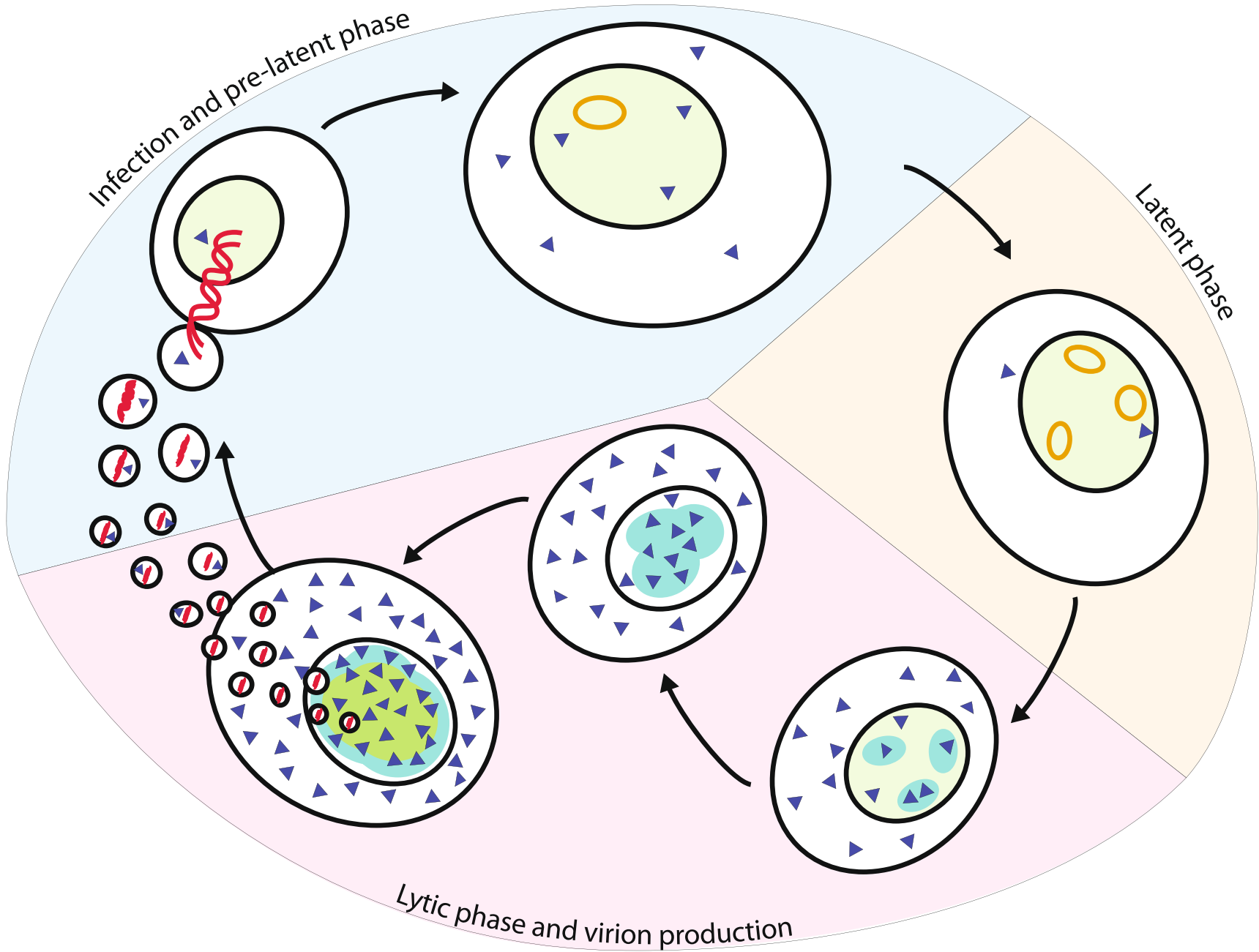

(1) virion navan

\section{$\checkmark \quad$ viral proteins}

\section{Epigenetically naïve viral DNA} replication compartment
Epigenetically marked viral DNA

inner replication compartment
Fig. 1 The tripartite life cycle of EBV. Infection and pre-latent phase (light blue segment). Upon infection, a virion releases it epigenetically naïve linear DNA (red) into its host cell. The viral DNA circularizes and makes its way into the nucleus (light green). As the incoming DNA is not epigenetically repressed, many viral proteins (blue triangles) are expressed at low levels initially. During this phase, the host cell grows in size and starts to proliferate (not shown). After a couple of days, histones and nucleosomes are positioned, and mostly repressive epigenetic marks are established on the viral, plasmid-like minichromosome. Latent phase (light yellow segment). EBV expresses only few latent EBV proteins to maintain and preserve its epigenetically repressed DNA in latently infected cells, and EBV DNA acquires a very high level of $\mathrm{CpG}$ methylation (not shown). Additionally, this strategy avoids the detection of viral antigens by the immune system. In the nucleus, several copies of viral repressed EBV minichromosomes accumulate via an unknown mechanism. Latent phase and virion production (light red segment). Upon reactivation of the virus' lytic phase, EBV starts to express lytic viral proteins, repressed viral chromatin opens up, and replication compartments (turquoise ovals) start to form. The full cascade of viral proteins is synthesized eventually, which counteracts the immune response of the host organism, supports lytic amplification of viral DNA, and provides structural proteins such as capsid components, tegument proteins, and glycoproteins. Replication compartments form, grow in dimension, and fuse during the massive replication of viral DNA. The newly synthesized viral DNA is packaged into viral capsid structures within the inner replication compartment (green area within the replication compartment). The newly replicated viral DNA is free of histones and lacks methylated $\mathrm{CpG}$ dinucleotides such that assembled virions contain epigenetically naïve viral DNA ready to infect new target cells. 
It is enigmatic what the initial steps of histone deposition on EBV DNA are and where the histones come from in the very early phase of infection. Canonical histones are produced only during the DNA synthesis (S) phase of the cell cycle, suggesting that the available histones might be noncanonical histone variants such as H3.3 or histones that belong to the small pool of free but chaperone-complexed, stored histones. Alternatively, histones are recycled in this early phase of viral infection, because the expression of two viral key activators, EBNA2 and EBNA-LP [58, 113], within the first $48 \mathrm{~h}$ of infection [74] induces global cellular gene expression (ibid.) that likely evicts nucleosomes from cellular chromatin. They might be available to be positioned onto the incoming naïve EBV DNA.

Clearly, histone chaperones meet the demand of de novo histone deposition to support diverse nuclear processes including the masking and gap-filling of EBV's naked genomic DNA. Among the many potential histone chaperones [47], HIRA, histone regulation A, a chaperone for the histone variant H3.3, seems to be an interesting candidate as it is involved in replication-independent histone deposition (together with DAXX and its co-chaperone ATRX). The HIRA complex has also been reported to act as gap filler strongly suggesting that it can interact with naked DNA [90]. In addition, HIRA is required for male pronucleus formation, which is inhibited due to a lack of nucleosome assembly in the sperm genome in the absence of HIRA [68]. HIRA is the only H3.3 chaperone that is incorporated broadly into decondensed sperm DNA at fertilization [68], which seems to be in line with the acquisition of a nucleosomal structure in EBV DNA.

Solid data are available for DAXX, also a chaperone for the histone variant H3.3, and its role in EBV infection. DAXX is bound by BNRF1, a tegument constituent and a member of a class of related proteins found in gamma herpesviruses [109] to promote selective viral gene expression [108, 110]. The binding of BNRF1 displaces the interaction of DAXX with the SWI/SNF-like chromatin remodeler ATRX [110]. ATRX directs DAXX to heterochromatin loci as ATRX specifically recognizes H3K9me3, a suppressive histone mark (see references in [47]). Surprisingly, virion-contained BNRF1 protein reduces the deposition of histone $\mathrm{H} 3.3$ onto viral DNA by DAXX and enhances viral gene expression early during EBV infection [50, 108]. This finding is interpreted to mean that BNRF1 prevents the deposition of histones and the formation of nucleosomes exclusively at promoters of EBV's latent genes that support B cell activation and transformation long term. It thus appears as if DAXX acts downstream after the initial formation of nucleosomes on EBV DNA to prevent epigenetic repression of critical latent genes of EBV.

Viral transcription appears to be pervasive early after infection in the pre-latent phase, because transcripts of lytic genes are found expressed although mostly at low levels. Among them is BZLF1 $[57,114]$ but also many others as revealed in a global transcriptomic approach ([74], see also the web tool http://ebv-b.helmholtz-muenchen.de/). It thus seems that the epigenetically naïve EBV DNA, which lacks histones and nucleosomes and is free of methylated $\mathrm{CpG}$ dinucleotides, serves as a permissive template for the cellular transcription machinery. This promiscuous state of transcription comes to an end presumably when EBV DNA acquires cellular nucleosomes, which restricts DNA accessibility, similar to other members of the herpesvirus family [2].

\section{Stable viral latency}

We know much about the epigenetic state of latent EBV infection in B cells. As a rule, several copies of EBV's genomic DNA are maintained as stable, extrachromosomal plasmids in latently infected cells ([26] for a recent review). The genome copies adopt a genuine epigenetic signature that is accomplished by the host cell. Viral latency is ensured by a strong epigenetic repression of lytic genes, while active latent viral genes are spared depending on the cell type. Repression is implemented and maintained by $\mathrm{CpG}$ methylation of viral DNA, by high-density packaging of nucleosomes, and by Polycomb group proteins that establish and maintain the key repressive modification $\mathrm{H} 3 \mathrm{~K} 27 \mathrm{me} 3$ on EBV chromatin [116]. The repressive $\mathrm{H} 3 \mathrm{~K} 9 \mathrm{me} 3$ histone mark is also detectable at low levels, but in contrast to $\mathrm{H} 3 \mathrm{~K} 27 \mathrm{me} 3$, H3K9me3 is not removed upon viral reactivation suggesting that this mark is not central to maintaining viral repressive chromatin (see the chapter "Lytic reactivation" below). The epigenetic signature of viral chromatin has been characterized and documented [7, 89, 106, 116].

CpG methylation of viral DNA is another layer of EBV's epigenetic regulation during latency. Surprisingly, the acquisition of $\mathrm{CpG}$ methylation is a very slow process in newly infected B-lymphocytes. Genomic EBV DNA in virions is virtually free of 5'-methylcytidine nucleotides, and $\mathrm{CpG}$ methylated residues are not detectable immediately after infection (Fig. S3 in [57]). The viral DNA slowly acquires cytidine methyl groups, a process that takes weeks to completion $[57,60]$. The slow kinetics suggest that $\mathrm{CpG}$ methylation of EBV DNA is not essential to downregulate lytic viral gene expression during the pre-latent phase of infection but might contribute to stabilize lytic gene repression long term. Clearly, EBV DNA reaches a very high level of $\mathrm{CpG}$ methylation, eventually, with a methylation rate of close to $100 \%$ in vitro in LCLs (Supplementary Fig. S1B and Table S1 in [116]) and in latently infected memory B cells in vivo (Fig. 1E in [116]). Most likely, de novo methyltransferases such as DNMT3a and $\mathrm{b}$ and the key maintenance methyltransferase DNMT1 introduce and maintain $\mathrm{CpG}$ methylation in genomic EBV DNA, 
respectively. How these enzymes are regulated during infection and how they get access to viral DNA are elusive.

Recent reports have studied the genomic organization of latent EBV genomes. The chromatin in different EBV cell lines and latency types differs [101], and also the three dimensional architecture of the EBV genome varies in cells in which the virus establishes different latency programs [7, 105]. The three-dimensional adjustment of the chromatin fiber is an important mechanism of gene regulation. Looping and bending of DNA and connecting or insulating cis-acting elements are also main principles of enhancer regulation. The ring-shaped cohesin complex is involved in the regulation of chromatin architecture in mammalian cells (summarized in [112]). The DNA-binding protein CTCF, the mediator complex, and the protein complex cohesin are central factors involved in these processes. Mediator and cohesin can connect enhancers to certain promoters over a long distance leading to their transcriptional activation [55]. Vice versa, CTCF and cohesion can insulate a promoter from an enhancer element promoting gene silencing $[80,115]$. Cohesin sites have recently been shown to be involved in the establishment of transcriptional memory by guiding transcription factors to their binding sites [118]. Moreover, cohesin has been also reported to act as a key structural element to regulate chromosome-wide gene expression [23].

Thus, it is not surprising that latent EBV chromatin is also organized via CTCF and cohesin subunits. There are close to twenty sites where CTCF and cohesin bind and were mapped in EBV's latent chromatin ([6, 7] and own unpublished findings), and certain CTCF sites have been implicated to act as insulators. They have been reported to prevent the spreading of repressive marks and progressive $\mathrm{CpG}$ methylation of viral DNA and keep latent viral promoters in an active state [106]. EBV's DNA is organized in loops [105] suggesting that higher-order chromosome conformations might be important to regulate levels of latent viral genes [67].

In the nucleus of latently infected cells, several copies of EBV's genomic DNA are maintained as stable, extrachromosomal plasmids, which replicate via oriP, the viral plasmid origin of DNA replication $([19,26]$ for two recent reviews). oriP is an important regulatory element that also acts as a viral enhancer to coordinate latent gene expression $[38,79]$. Its function is under the control of EBNA1, a latent viral factor that binds oriP and likely ensures prominent nucleosome-free or poor regions within two parts of oriP, the family or repeats and the dyad symmetry element $[7,72]$. As a consequence, neighboring nucleosomes contain histones with activating marks such as H3K4me3, H3K27ac, and H3K4me1 (unpublished data) suggesting that oriP is an island of euchromatin to support transcription of latent genes such as the latent membrane proteins and several EBNAs in an otherwise heterochromatic and epigenetically repressed EBV genome. Interestingly, oriP also mediates a molecular link to host cell chromosomes suggesting that it acts as an anchor to tether EBV's genomic copies to nuclear chromatin [73]. Again, EBNA1 is the critical viral factor in trans. In latently EBVinfected cells, it mediates tethering of viral DNA to cellular perichromatin [28] and ensures the maintenance and partitioning of viral genome copies in resting and proliferating cells, respectively [19].

\section{Lytic reactivation}

In the latent phase, all lytic genes are strictly repressed, but the ectopic expression of a single viral gene, BZLF1 (also called EB1, ZEBRA, Z, or Zta), can induce the full lytic cycle in certain cells in vitro $[21,102]$. In latently infected $B$ cells in vivo, BZLF1 is expressed when memory B cells encounter their cognate antigens and terminally differentiate into plasma cells, a process that supports virus de novo synthesis [62, 107]. Also, in vitro activation of the B cell receptor is clearly linked to viral reactivation in certain established cell lines latently infected with EBV $([4,98]$ for earlier reviews $[111$, 121] for recently published work). Viral micro RNAs counterregulate downstream signals of the $\mathrm{B}$ cell receptor and interfere with lytic phase induction suggesting that $B$ cell receptor triggering is an important route to escape from latency [17]. The regulation of BZLF1 transcription downstream of the activating signaling cascade is complex and involves numerous cellular transcriptional activators and repressors, chromatin constituents, and histone modifications to activate the repressed BZLF1 gene. Regulated expression of BZLF1 has been the subject of several recent reviews $[37,59,76]$ and recent original work [69]. Not surprisingly, repression of BZLF1's promoter appears similarly complex and multifaceted. The structure and epigenetic landscape of the BZLF1 promoter differ from the majority of EBV's lytic promoters of the so-called early class of viral genes as it contains only very few $\mathrm{CpG}$ dinucleotides, which are even spared from cytosine methylation in vitro and partially also in vivo [116] suggesting that BZLF1's repression is probably not controlled by $\mathrm{CpG}$ methylation.

Upon induced reactivation or ectopic BZLF1 expression, the second essential early lytic gene, BRLF1, is expressed. Both genes are indispensable for the lytic phase [33] and encode transactivators that activate viral and certain cellular promoters, leading to an ordered cascade of viral gene expression: activation of early gene expression followed by the lytic cascade of viral genome replication and late gene expression.

\section{BZLF1 is EBV's lytic switch}

Much is known about BZLF1 and its molecular functions. Briefly, BZLF1 is a member of the AP-1 family and a bZIP 
protein, binds viral and cellular DNA sequence specifically as a homodimer and even in nucleosome-dense compacted chromatin, activates transcription, acts as a viral pioneer factor, recruits chromatin remodelers that open repressed chromatin locally where BZLF1 binds, has a peculiar preference to bind to $\mathrm{CpG}$ methylated DNA sequence motifs, and is indispensable for lytic viral DNA replication as it recruits viral replication factors to EBV's lytic origin of DNA replication, oriLyt. Many of BZLF1's molecular functions have been detailed, recently.

The atomic structure of BZLF1 bound to DNA has been resolved $[49,83]$. It shows the binding of BZLF1 to two different classes of related sequence motifs $[8,9,32,119]$ with two rather different dissociation constants [8]. Paradoxically, BZLF1 preferentially binds to methylated-binding motifs, commonly called meZREs, such as 5'-TGAGmeCGA-3', which are prevalent in highly $\mathrm{CpG}$ methylated promoters of viral early genes in EBV DNA. These sequence elements contain two 5'-methylcytosines $(\mathrm{meC})$ in the top and bottom strands. BZLF1 was the first example of a sequence-specific transcription factor that preferentially recognizes and selectively binds methylated cytosine residues within a specific sequence. This exceptional feature is essential for this herpesvirus to escape from its latent phase of infection $[56,57]$.

Upon induction in latently infected cells, BZLF1 induces downstream viral promoters of several early genes some of which encode essential components of the autonomous DNA replication machinery of EBV's lytic phase [34, 35]. The promoters typically encompass $\mathrm{CpG}$ dinucleotides in high density and clusters of meZRE sites, which have to be methylated to be bound by BZLF1 to induce these early genes $[8,56,57$, $87,116,119]$. At low levels, BZLF1 stably binds to meZREs in viral and cellular chromatin in contrast to non-meZREs sites that BZLF1 only binds efficiently at high BZLF1 levels [8, 14]. For example, the promoters of the early lytic BBLF4 and BBLF2/3 genes, which are both essential for lytic viral DNA amplification [34, 35], contain numerous meZREs, which are preferentially bound when methylated. Only then can BZLF1 activate these promoters efficiently [8].

BZLF1 also needs to bind certain low affinity sites in the viral lytic origin of DNA replication, oriLyt, where BZLF1 acts as an essential replication factor [94, 95]. This aspect suggests that BZLF1 has to reach a considerably high threshold level to support EBV's lytic phase fully (see below). Moreover, expression of many late viral genes depends on amplified genomic DNA templates that result from lytic viral DNA replication only ([15, 29, 41]; reviewed in [42]). It thus seems that critical BZLF1 levels directly and indirectly control the ordered and timely lytic expression of all viral genes. Autoregulation of BZLF1 expression via a positive feedback loop [98] and nonmeZRE sites and meZRE sites (in the promoters of BZLF1 and BRLF1, respectively) might be a prerequisite to achieve sufficiently high BZLF1 levels to complete EBV's lytic phase [10, 36, 98].

Recently, characterized molecular functions of BZLF1 suggest that this viral protein is a bona fide pioneer transcription factor [120] that has direct access to epigenetically repressed (viral) chromatin inducing its transcriptional competence. Upon sequence-specific binding even in densely packed nucleosomal EBV DNA [93], BZLF1 unfolds its potential as a pioneer factor preferentially on meZRE sites [116]. BZLF1 recruits chromatin remodelers such as INO80 and probably other abundant remodelers that evict local nucleosomes providing access to promoters and cis-regulatory elements in viral chromatin [93]. As a consequence, nucleosomes at BZLF1binding sites are lost, and repressive chromatin marks such as $\mathrm{H} 3 \mathrm{~K} 27 \mathrm{me} 3$ on flanking histones are erased, while $\mathrm{H} 3 \mathrm{~K} 9 \mathrm{me} 3$ marks are not affected $[75,89,116]$. Concomitantly, previously bound Polycomb group proteins (PCG) and the writer EZH2 are no longer associated with viral DNA [116]. Induction of EBV's lytic phase eliminates the repressive H3K27me3 mark on lytic promoters relieving their tight repression. The resulting open chromatin allows loading of the RNA polymerase II (RNAP II) multiprotein complex with its transcriptional machinery. Active histone marks such as H3K4me3 are set especially at early lytic promoters [116] and trigger EBV's escape from latency. Additionally, the interaction of BZLF1 with the histone acetyltransferase CBP increases the transactivation of early EBV promoters [1, 27]. The subsequent acetylation of histones in the promoters of BRLF1 and BZLF1 correlates with their expression [16, 52, $75]$ but is not necessarily sufficient to induce them $[22,40]$. Unexpectedly, CpG methylation of viral DNA is maintained throughout this early viral phase of transcriptional reactivation and is no hindrance to active transcription of extensively $\mathrm{CpG}$ methylated viral genes as thought previously [116].

In contrast to the consequences of BZLF1 binding to viral DNA, alteration of the chromatin architecture of EBV's genomic DNA and impaired functions of the chromatin organizer CTCF, which organizes the viral genome during latency and regulates the viral programs of latent transcription ([82] for a recent review), do not result in lytic reactivation [69].

Upon lytic reactivation, the EBV minichromosomes move within the nucleus from their pericentric positions [28] with gene-poor, AT-rich, and repressive heterochromatin to active euchromatin [73]. EBV's genomic DNA remains attached to the nuclear matrix as during the latent phase of infection, but the point of attachment changes upon lytic reactivation from the plasmid origin of DNA replication oriP [51] to oriLyt sequences [71]. It seems as if EBV positions its genomes in a higher-order nuclear context reflecting its requirements for efficient expression of its genes.

EBV not only governs the cellular machinery to reverse epigenetic repression of the viral genome but also induces a genome-wide reorganization and alteration of the cellular 
epigenome and transcriptome. As in viral DNA, BZLF1 binds the same two major sequence motifs in cellular chromatin [14, 88]. More than 190,000 $( \pm 40,000)$ BZLF1-binding sites are identified [14] throughout cellular DNA [81, 104]. While the induced expression of BZLF1 in EBV-negative cells causes only minor alterations of cellular gene expression, the expression of BZLF1 in latently infected B cells results in a massive reduction of the cellular transcriptome and profoundly alters the cellular epigenome within 6 to $15 \mathrm{~h}$ post induction [14]. Regions of previously open and accessible chromatin close genome-wide. Concomitantly with the reduction of open chromatin, middle- and long-distance chromatin interactions between promoters and their interacting regulatory regions are strongly reduced [14]. EBV's objective of these alterations is not understood in the moment, but already Adamson et al. speculated in 1999 that the interaction of BZLF1 with CBP might interfere with the availability of histone acetyltransferase CBP at transcriptionally active cellular loci [1]. Within 1 day of lytic phase induction, cellular chromatin was found to become highly condensed, the nuclear lamina was redistributed [63], and cellular histones appear to move to the periphery of the cell nucleus [18].

The purpose of restructuring cellular chromatin is not obvious but might have its origin in creating space for nuclear sites where lytic amplification of viral DNA takes place. The nuclear structures that are termed amplification or replication factories (or compartments) [63] form in lytically induced cells infected with EBV [104] or other viruses (reviewed in [96]). Viral proteins known to associate with the lytic replication of viral DNA accumulate in EBV's replication compartments, which are microscopically visible structures (Fig. 1 in [24]) where viral DNA replicates and amplifies massively [78].

\section{Loss of chromatin constituents and DNA methylation upon lytic amplification of viral DNA}

The replication compartments appear within the nucleus seeded by single viral genomes [78] and increase in size over time, fuse, and finally occupy large parts of the nucleus (Fig. 1 in [24]). When EBV's DNA is amplified in the replication compartments and becomes microscopically detectable, it does not co-localize with histone chaperones nor with canonical or noncanonical histones such as H2B, H3.1, or H3.3 [18] indicating that cellular machines that deposits histones on newly synthesized cellular DNA are excluded from viral replication compartments. Lack of nucleosomal structures alters the superhelicity of DNA, and in fact, viral DNA was found to be less supercoiled in this phase compared to DNA extracted from latently infected cells [84] supporting this view.
Viral replication compartments are operationally subdivided into outer ["ongoing replication foci"] and inner ["BMRF1-cores"] domains [100], in which early and late lytic genes are transcribed, respectively [99], while transcription of late lytic genes is only conducted simultaneously with the replication of and from newly synthesized viral genomes free of nucleosomes ([29, 30, 64]; reviewed in [15]). While these findings might give the impression that unmethylated viral DNA is required for late lytic gene expression, demethylation of viral DNA alone is not sufficient [64]. The molecular link between herpesvirus lytic DNA replication and late gene transcription is interesting and has been reviewed very recently $[15,42]$.

Within the outer domain of replication compartments, ongoing de novo viral DNA synthesis is accompanied by cellular homologous recombination repair proteins, while cellular mismatch repair proteins were found in the inner domain, where the newly synthesized DNA seems to accumulate [25, $61,100]$.

Also excluded from lytically replicating herpesviral DNA in replication compartments is proliferating cell nuclear antigen (PCNA) [77]. PCNA is essential for eukaryotic licensed DNA replication (and DNA repair) and acts as a trimeric DNA sliding clamp and processivity factor for DNA polymerase $\delta$ in eukaryotic cells and as a scaffold to recruit proteins involved in DNA replication, DNA repair, chromatin remodeling, and epigenetics. Among the recruited proteins is the cellular DNA methyltransferase 1, DNMT1, that together with UHFR1 couple semiconservative cellular DNA replication and DNA methylation such that newly replicated daughter strands inherit the pattern of $\mathrm{CpG}$ methylation of parental DNA. In cells that support EBV's lytic phase, PCNA localizes to the amplification factories, but PCNA is not detected at the sites of viral DNA synthesis [18]. This is no problem for the virus because it encodes its own autonomous viral factors that mediate lytic herpesviral DNA replication independent of the host cell $[34,35]$. The function of PCNA in lytic DNA replication of EBV is replaced by the structural homolog BMRF1 [46]. In the absence of PCNA, DNMT1 is not recruited to the replication forks of herpesviral DNA, and methylation marks at cytosine residues are lost on both newly synthesized DNA strands, removing this epigenetic modification from EBV's genomic DNA. To our knowledge, this scenario has not been formally proven yet, but it is highly plausible ([18, 57] and references therein). As a consequence, the isolated packaging of the amplified viral DNA into capsids ensures that EBV's genomic DNA within virions is free of methylated CpG dinucleotides ([60], Supplementary Fig. S3 in ref. [57]).

In summary, EBV prevents the loading of cellular histones on its newly synthesized DNA [54] and uncouples viral DNA replication from the activity of DNMT1 that maintains the methylation pattern in the newly synthesized strand in cellular DNA [56]. Both strategies ensure that EBV's DNA in virions 
is epigenetically naïve when the virus launches its next epigenetic life cycle in a newly infected cell. Surprisingly, BZLF1 will be detectably expressed in the newly infected cell within hours $[44,57,114]$, presumably because the epigenetically naked viral DNA does not prevent an initial, promiscuous, and genome-wide transcription [53]. Nevertheless, BZLF1's transient expression fails to initiate EBV's lytic phase in these newly infected cells. The lack of CpG methylation of EBV's virion DNA seems to be an important reason because unmethylated meZRE sequence motifs will not allow an efficient BZLF1 binding preventing the onset of the cascade of lytic viral genes from the start [117]. The abundance of cellular BZLF1-binding sites might be a second fail-safe mode to mitigate the accidental induction of EBV's lytic phase in freshly infected cells [14] indicating that latency is definitely EBV's preferred lifestyle.

\section{Open questions}

\section{Chromatin acquisition when, how, and by whom}

It is unknown what drives the very rapid nucleosome formation on the incoming EBV DNA very early after infection of primary human B-lymphocytes. There are assumed players such as histone chaperones, but this area is rather speculative. It might be rewarding to invest here, because this viral model offers an attractive, perhaps even unique, opportunity to investigate de novo chromatin assembly and regulation in a tractable system and in mammalian cells. The epigenetic mechanisms of developmental programming during fertilization in the zygotic state and in the early embryo are technically difficult to study [13], but the EBV infection model is biochemically and genetically accessible, which seems to be attractive.

\section{Polycomb recruitment and timing of H3K27me3 histone marks}

It remains elusive how EBV DNA acquires nucleosomes and when they turn into repressed chromatin as indicated by characteristic H3K27me3 histone marks (among others). Similar to EBV, its closest relative, Kaposi sarcoma-associated herpesvirus (KSHV), can also establish latent infections in certain cell types. Recently, in a collaborative work, Adam Grundhoff has studied the kinetics of Polycomb repressive complexes (PRC) on KSHV DNA in infected cells and found a correlation between the recruitment of the noncanonical PRC1.1 component KDM2B, CpG islands, and H3K27me3 marks suggesting that high local concentrations of unmethylated $\mathrm{CpG}$ clusters attract Polycomb complexes to implement chromatin repression on viral DNA [43]. KSHV differs from EBV as KSVH chromatin adopts a bivalent mode in its latent phase.
Therefore, it remains to be investigated how and when EBV's DNA acquires typical repressive marks indicative of Polycomb functions.

\section{Epigenetics and EBV-associated malignancies}

EBV is associated with or even causes very different types of malignancies in man. Latent viral gene products are likely contributors to oncogenic processes in these tumor cells, but also lytic EBV genes have been blamed to be involved in carcinogenesis. Even in tumors that are predominantly latently infected such as nasopharyngeal carcinoma, for example, fractions of cells enter the lytic phase perhaps playing a contributing role to tumor formation $[66,70]$. Immune responses to both latent and lytic viral gene products are manifold, broad, and rather diverse, but the optional induction of the lytic phase in tumors cells likely turns them into better targets for cellular immunity [66]. It is intriguing to think that the manipulation of the epigenetic program in EBV tumors in vivo might provide a therapeutic window as discussed recently [65].

Funding information Open Access funding provided by Projekt DEAL.

\section{Compliance with ethical standards}

Conflict of interest The authors declare that they have no conflict of interest.

Open Access This article is licensed under a Creative Commons Attribution 4.0 International License, which permits use, sharing, adaptation, distribution and reproduction in any medium or format, as long as you give appropriate credit to the original author(s) and the source, provide a link to the Creative Commons licence, and indicate if changes were made. The images or other third party material in this article are included in the article's Creative Commons licence, unless indicated otherwise in a credit line to the material. If material is not included in the article's Creative Commons licence and your intended use is not permitted by statutory regulation or exceeds the permitted use, you will need to obtain permission directly from the copyright holder. To view a copy of this licence, visit http://creativecommons.org/licenses/by/4.0/.

\section{References}

1. Adamson AL, Kenney S (1999) The Epstein-Barr virus BZLF1 protein interacts physically and functionally with the histone acetylase CREB-binding protein. J Virol 73:6551-6558

2. Albright ER, Kalejta RF (2016) Canonical and variant forms of histone $\mathrm{H} 3$ are deposited onto the human cytomegalovirus genome during lytic and latent infections. J Virol 90:10309-10320. https://doi.org/10.1128/JVI.01220-16

3. Amon W, Binne UK, Bryant H, Jenkins PJ, Karstegl CE, Farrell PJ (2004) Lytic cycle gene regulation of Epstein-Barr virus. J Virol 78: 13460-13469. https://doi.org/10.1128/JVI.78.24.13460-13469.2004

4. Amon W, Farrell PJ (2005) Reactivation of Epstein-Barr virus from latency. Rev Med Virol 15:149-156. https://doi.org/10. 1002/rmv.456 
5. Anonymous (1997) Epstein-Barr virus; IARC monographs on the evaluation of carcinogenic risks to humans. IARC, Lyon, $p 1$

6. Arvey A, Tempera I, Lieberman PM (2013) Interpreting the Epstein-Barr virus (EBV) epigenome using high-throughput data. Viruses. 5:1042-1054. https://doi.org/10.3390/v5041042

7. Arvey A, Tempera I, Tsai K, Chen HS, Tikhmyanova N, Klichinsky M, Leslie C, Lieberman PM (2012) An atlas of the Epstein-Barr virus transcriptome and epigenome reveals hostvirus regulatory interactions. Cell Host Microbe 12:233-245. https://doi.org/10.1016/j.chom.2012.06.008

8. Bergbauer M, Kalla M, Schmeinck A, Gobel C, Rothbauer U, Eck S, Benet-Pages A, Strom TM, Hammerschmidt W (2010) CpGmethylation regulates a class of Epstein-Barr virus promoters. PLoS Pathog 6:e1001114. https://doi.org/10.1371/journal.ppat. 1001114

9. Bhende PM, Seaman WT, Delecluse HJ, Kenney SC (2004) The EBV lytic switch protein, Z, preferentially binds to and activates the methylated viral genome. Nat Genet 36:1099-1104. https:// doi.org/10.1038/ng1424

10. Binne UK, Amon W, Farrell PJ (2002) Promoter sequences required for reactivation of Epstein-Barr virus from latency. J Virol 76:10282-10289

11. Bloss TA, Sugden B (1994) Optimal lengths for DNAs encapsidated by Epstein-Barr virus. J Virol 68:8217-8222

12. Brykczynska U, Hisano M, Erkek S, Ramos L, Oakeley EJ, Roloff TC, Beisel C, Schübeler D, Stadler MB, Peters AH (2010) Repressive and active histone methylation mark distinct promoters in human and mouse spermatozoa. Nat Struct Mol Biol 17:679687. https://doi.org/10.1038/nsmb.1821

13. Burton A, Torres-Padilla ME (2014) Chromatin dynamics in the regulation of cell fate allocation during early embryogenesis. Nat Rev Mol Cell Biol 15:723-734. https://doi.org/10.1038/nrm3885

14. Buschle A, Mrozek-Gorska P, Krebs S, Blum H, Cernilogar FM, Schotta G, Pich D, Straub T, and Hammerschmidt W. 2019. Epstein-Barr virus inactivates the transcriptome and disrupts the chromatin architecture of its host cell in the first phase of lytic reactivation. biorxiv.org. bioRxiv 573659. https://doi.org/10. $1101 / 573659$

15. Chakravorty A, Sugden B, Johannsen EC (2019) An epigenetic journey: Epstein-Barr virus transcribes chromatinized and subsequently unchromatinized templates during its lytic cycle. J Virol 93:e2247-e02218. https://doi.org/10.1128/JVI.02247-18

16. Chang LK, Liu ST (2000) Activation of the BRLF1 promoter and lytic cycle of Epstein-Barr virus by histone acetylation. Nucleic Acids Res 28:3918-3925. https://doi.org/10.1093/nar/28.20.3918

17. Chen Y, Fachko D, Ivanov NS, Skinner CM, Skalsky RL (2019) Epstein-Barr virus microRNAs regulate B cell receptor signal transduction and lytic reactivation. PLoS Pathog 15:e1007535. https://doi.org/10.1371/journal.ppat.1007535

18. Chiu YF, Sugden AU, Sugden B (2013) Epstein-Barr viral productive amplification reprograms nuclear architecture, DNA replication, and histone deposition. Cell Host Microbe 14:607-618. https://doi.org/10.1016/j.chom.2013.11.009

19. Chiu YF, Sugden B (2018) Plasmid partitioning by human tumor viruses. J Virol 92:e02170-e02117. https://doi.org/10.1128/JVI. 02170-17

20. Cohen JI, Fauci AS, Varmus H, Nabel GJ (2011) Epstein-Barr virus: an important vaccine target for cancer prevention. Sci Transl Med 3:107fs7. https://doi.org/10.1126/scitranslmed. 3002878

21. Countryman J, Jenson H, Seibl R, Wolf H, Miller G (1987) Polymorphic proteins encoded within BZLF1 of defective and standard Epstein-Barr viruses disrupt latency. J Virol 61:36723679

22. Countryman JK, Gradoville L, Miller G (2008) Histone hyperacetylation occurs on promoters of lytic cycle regulatory genes in Epstein-Barr virus-infected cell lines which are refractory to disruption of latency by histone deacetylase inhibitors. J Virol 82:4706-4719. https://doi.org/10.1128/JVI.00116-08

23. Crane E, Bian Q, McCord RP, Lajoie BR, Wheeler BS, Ralston EJ, Uzawa S, Dekker J, Meyer BJ (2015) Condensin-driven remodelling of $\mathrm{X}$ chromosome topology during dosage compensation. Nature. 523:240-244. https://doi.org/10.1038/nature14450

24. Daikoku T, Kudoh A, Fujita M, Sugaya Y, Isomura H, Shirata N, Tsurumi T (2005) Architecture of replication compartments formed during Epstein-Barr virus lytic replication. J Virol 79: 3409-3418. https://doi.org/10.1128/JVI.79.6.3409-3418.2005

25. Daikoku T, Kudoh A, Sugaya Y, Iwahori S, Shirata N, Isomura H, Tsurumi T (2006) Postreplicative mismatch repair factors are recruited to Epstein-Barr virus replication compartments. J Biol Chem 281:11422-11430. https://doi.org/10.1074/jbc. M510314200

26. De Leo A, Calderon A, Lieberman PM (2019) Control of viral latency by episome maintenance proteins. Trends Microbiol. https://doi.org/10.1016/j.tim.2019.09.002

27. Deng Z, Chen CJ, Chamberlin M, Lu F, Blobel GA, Speicher D, Cirillo LA, Zaret KS, Lieberman PM (2003) The CBP bromodomain and nucleosome targeting are required for Ztadirected nucleosome acetylation and transcription activation. Mol Cell Biol 23:2633-2644. https://doi.org/10.1128/mcb.23.8. 2633-2644.2003

28. Deutsch MJ, Ott E, Papior P, Schepers A (2010) The latent origin of replication of Epstein-Barr virus directs viral genomes to active regions of the nucleus. J Virol 84:2533-2546. https://doi.org/10. 1128/JVI.01909-09

29. Djavadian R, Chiu YF, Johannsen E (2016) An Epstein-Barr virus-encoded protein complex requires an origin of lytic replication in cis to mediate late gene transcription. PLoS Pathog 12: e1005718. https://doi.org/10.1371/journal.ppat.1005718

30. Djavadian R, Hayes M, Johannsen E (2018) CAGE-seq analysis of Epstein-Barr virus lytic gene transcription: 3 kinetic classes from 2 mechanisms. PLoS Pathog 14:e1007114. https://doi.org/ 10.1371/journal.ppat.1007114

31. Ersing I, Nobre L, Wang LW, Soday L, Ma Y, Paulo JA, Narita Y, Ashbaugh CW, Jiang C, Grayson NE, Kieff E, Gygi SP, Weekes MP, Gewurz BE (2017) A temporal proteomic map of EpsteinBarr virus lytic replication in B cells. Cell Rep 19:1479-1493. https://doi.org/10.1016/j.celrep.2017.04.062

32. Farrell PJ, Rowe DT, Rooney CM, Kouzarides T (1989) EpsteinBarr virus BZLF1 trans-activator specifically binds to a consensus AP-1 site and is related to c-fos. EMBO J 8:127-132

33. Feederle R, Kost M, Baumann M, Janz A, Drouet E, Hammerschmidt W, Delecluse HJ (2000) The Epstein-Barr virus lytic program is controlled by the co-operative functions of two transactivators. EMBO J 19:3080-3089. https://doi.org/10.1093/ emboj/19.12.3080

34. Fixman ED, Hayward GS, Hayward SD (1992) Trans-acting requirements for replication of Epstein-Barr virus ori-Lyt. J Virol 66: 5030-5039

35. Fixman ED, Hayward GS, Hayward SD (1995) Replication of Epstein-Barr virus oriLyt: lack of a dedicated virally encoded origin-binding protein and dependence on $\mathrm{Zta}$ in cotransfection assays. J Virol 69:2998-3006

36. Flemington E, Speck SH (1990) Autoregulation of Epstein-Barr virus putative lytic switch gene BZLF1. J Virol 64:1227-1232

37. Frost TC, Gewurz BE (2018) Epigenetic crossroads of the Epstein-Barr virus B-cell relationship. Curr Opin Virol 32:1523. https://doi.org/10.1016/j.coviro.2018.08.012

38. Gahn TA, Sugden B (1995) An EBNA-1-dependent enhancer acts from a distance of 10 kilobase pairs to increase expression of the Epstein-Barr virus LMP gene. J Virol 69:2633-2636 
39. Gibson W, Roizman B (1971) Compartmentalization of spermine and spermidine in the herpes simplex virion. Proc Natl Acad Sci U S A 68:2818-2821

40. Gradoville L, Kwa D, El-Guindy A, Miller G (2002) Protein kinase $\mathrm{C}$-independent activation of the Epstein-Barr virus lytic cycle. J Virol 76:5612-5626. https://doi.org/10.1128/jvi.76.11.56125626.2002

41. Gruffat H, Kadjouf F, Mariame B, Manet E (2012) The EpsteinBarr virus BcRF1 gene product is a TBP-like protein with an essential role in late gene expression. J Virol 86:6023-6032. https://doi.org/10.1128/JVI.00159-12

42. Gruffat H, Marchione R, Manet E (2016) Herpesvirus late gene expression: a viral-specific pre-initiation complex is key. Front Microbiol 7:869. https://doi.org/10.3389/fmicb.2016.00869

43. Günther T, Fröhlich J, Herrde C, Ohno S, Burkhardt L, Adler H, Grundhoff A (2019) A comparative epigenome analysis of gammaherpesviruses suggests cis-acting sequence features as critical mediators of rapid polycomb recruitment. PLoS Pathog 15: e1007838. https://doi.org/10.1371/journal.ppat.1007838

44. Halder S, Murakami M, Verma SC, Kumar P, Yi F, Robertson ES (2009) Early events associated with infection of Epstein-Barr virus infection of primary B-cells. PLoS One 4:e7214. https://doi.org/ 10.1371/journal.pone.0007214

45. Hammerschmidt W (2015) The epigenetic life cycle of EpsteinBarr virus. Curr Top Microbiol Immunol 390:103-117. https://doi. org/10.1007/978-3-319-22822-8 6

46. Hammerschmidt W, Sugden B (2013) Replication of Epstein-Barr viral DNA. Cold Spring Harb Perspect Biol 5:513-525. https:// doi.org/10.1101/cshperspect.a013029

47. Hammond CM, Strømme CB, Huang H, Patel DJ, Groth A (2017) Histone chaperone networks shaping chromatin function. Nat Rev Mol Cell Biol 18:141-158. https://doi.org/10.1038/nrm.2016.159

48. Henle W, Henle G, Zajac BA, Pearson G, Waubke R, Scriba M (1970) Differential reactivity of human serums with early antigens induced by Epstein-Barr virus. Science. 169:188-190. https://doi. org/10.1126/science.169.3941.188

49. Hong S, Wang D, Horton JR, Zhang X, Speck SH, Blumenthal RM, Cheng X (2017) Methyl-dependent and spatial-specific DNA recognition by the orthologous transcription factors human AP-1 and Epstein-Barr virus Zta. Nucleic Acids Res 45:2503-2515. https://doi.org/10.1093/nar/gkx057

50. Huang H, Deng Z, Vladimirova O, Wiedmer A, Lu F, Lieberman PM, Patel DJ (2016) Structural basis underlying viral hijacking of a histone chaperone complex. Nat Commun 7:12707. https://doi. org/10.1038/ncomms 12707

51. Jankelevich S, Kolman JL, Bodnar JW, Miller G (1992) A nuclear matrix attachment region organizes the Epstein-Barr viral plasmid in Raji cells into a single DNA domain. EMBO J 11:1165-1176

52. Jenkins PJ, Binne UK, Farrell PJ (2000) Histone acetylation and reactivation of Epstein-Barr virus from latency. J Virol 74:710 720. https://doi.org/10.1128/jvi.74.2.710-720.2000

53. Jochum S, Ruiss R, Moosmann A, Hammerschmidt W, Zeidler R (2012) RNAs in Epstein-Barr virions control early steps of infection. Proc Natl Acad Sci U S A 109:E1396-E1404. https://doi.org/ 10.1073/pnas.1115906109

54. Johannsen E, Luftig M, Chase MR, Weicksel S, Cahir-McFarland E, Illanes D, Sarracino D, Kieff E (2004) Proteins of purified Epstein-Barr virus. Proc Natl Acad Sci U S A 101:1628616291. https://doi.org/10.1073/pnas.0407320101

55. Kagey MH, Newman JJ, Bilodeau S, Zhan Y, Orlando DA, van Berkum NL, Ebmeier CC, Goossens J, Rahl PB, Levine SS, Taatjes DJ, Dekker J, Young RA (2010) Mediator and cohesin connect gene expression and chromatin architecture. Nature. 467:430-435. https://doi.org/10.1038/nature09380

56. Kalla M, Gobel C, Hammerschmidt W (2012) The lytic phase of Epstein-Barr virus requires a viral genome with 5-methylcytosine residues in CpG sites. J Virol 86:447-458. https://doi.org/10.1128/ JVI.06314-11

57. Kalla M, Schmeinck A, Bergbauer M, Pich D, Hammerschmidt W (2010) AP-1 homolog BZLF1 of Epstein-Barr virus has two essential functions dependent on the epigenetic state of the viral genome. Proc Natl Acad Sci U S A 107:850-855. https://doi. org/10.1073/pnas.0911948107

58. Kempkes B, Ling PD (2015) EBNA2 and its coactivator EBNALP. Curr Top Microbiol Immunol 391:35-59. https://doi.org/10. 1007/978-3-319-22834-1 2

59. Kenney SC, Mertz JE (2014) Regulation of the latent-lytic switch in Epstein-Barr virus. Semin Cancer Biol 26:60-68. https://doi. org/10.1016/j.semcancer.2014.01.002

60. Kintner C, Sugden B (1981) Conservation and progressive methylation of Epstein-Barr viral DNA sequences in transformed cells. J Virol 38:305-316

61. Kudoh A, Iwahori S, Sato Y, Nakayama S, Isomura H, Murata T, Tsurumi T (2009) Homologous recombinational repair factors are recruited and loaded onto the viral DNA genome in Epstein-Barr virus replication compartments. J Virol 83:6641-6651. https://doi. org/10.1128/JVI.00049-09

62. Laichalk LL, Thorley-Lawson DA (2005) Terminal differentiation into plasma cells initiates the replicative cycle of Epstein-Barr virus in vivo. J Virol 79:1296-1307. https://doi.org/10.1128/JVI. 79.2.1296-1307.2005

63. Lee CP, Chen JY, Wang JT, Kimura K, Takemoto A, Lu CC, Chen MR (2007) Epstein-Barr virus BGLF4 kinase induces premature chromosome condensation through activation of condensin and topoisomerase II. J Virol 81:5166-5180. https://doi.org/10.1128/ JVI.00120-07

64. Li D, Fu W, Swaminathan S (2018a) Continuous DNA replication is required for late gene transcription and maintenance of replication compartments in gammaherpesviruses. PLoS Pathog 14: e1007070. https://doi.org/10.1371/journal.ppat.1007070

65. Li H, Hu J, Luo X, Bode AM, Dong Z, Cao Y (2018b) Therapies based on targeting Epstein-Barr virus lytic replication for EBVassociated malignancies. Cancer Sci 109:2101-2108. https://doi. org/10.1111/cas.13634

66. Li H, Liu S, Hu J, Luo X, Li N, Bode AM, Cao Y (2016) EpsteinBarr virus lytic reactivation regulation and its pathogenic role in carcinogenesis. Int J Biol Sci 12:1309-1318. https://doi.org/10. $7150 /$ ijbs. 16564

67. Lieberman PM (2015) Chromatin structure of Epstein-Barr virus latent episomes. Curr Top Microbiol Immunol 390:71-102. https://doi.org/10.1007/978-3-319-22822-8 5

68. Lin CJ, Koh FM, Wong P, Conti M, Ramalho-Santos M (2014) Hira-mediated H3.3 incorporation is required for DNA replication and ribosomal RNA transcription in the mouse zygote. Dev Cell 30:268-279. https://doi.org/10.1016/j.devcel.2014.06.022

69. Lupey-Green LN, Moquin SA, Martin KA, McDevitt SM, Hulse M, Caruso LB, Pomerantz RT, Miranda JL, Tempera I (2017) PARP1 restricts Epstein Barr virus lytic reactivation by binding the BZLF1 promoter. Virology. 507:220-230. https://doi.org/10. 1016/j.virol.2017.04.006

70. Manners O, Murphy JC, Coleman A, Hughes DJ, Whitehouse A (2018) Contribution of the KSHV and EBV lytic cycles to tumourigenesis. Curr Opin Virol 32:60-70. https://doi.org/10. 1016/j.coviro.2018.08.014

71. Mattia E, Ceridono M, Chichiarelli S, D'Erme M (1999) Interactions of Epstein-Barr virus origins of replication with nuclear matrix in the latent and in the lytic phases of viral infection. Virology. 262:9-17

72. McClellan MJ, Wood CD, Ojeniyi O, Cooper TJ, Kanhere A, Arvey A, Webb HM, Palermo RD, Harth-Hertle ML, Kempkes B, Jenner RG, West MJ (2013) Modulation of enhancer looping and differential gene targeting by Epstein-Barr virus transcription 
factors directs cellular reprogramming. PLoS Pathog 9:e1003636. https://doi.org/10.1371/journal.ppat.1003636

73. Moquin SA, Thomas S, Whalen S, Warburton A, Fernandez SG, McBride AA, Pollard KS, Miranda JJL (2017) The Epstein-Barr virus episome maneuvers between nuclear chromatin compartments during reactivation. J Virol. https://doi.org/10.1128/JVI. 01413-17

74. Mrozek-Gorska P, Buschle A, Pich D, Schwarzmayr T, Fechtner R, Scialdone A, Hammerschmidt W (2019) Epstein-Barr virus reprograms human B lymphocytes immediately in the prelatent phase of infection. Proc Natl Acad Sci U S A 116:16046-16055. https://doi.org/10.1073/pnas.1901314116

75. Murata T, Kondo Y, Sugimoto A, Kawashima D, Saito S, Isomura H, Kanda T, Tsurumi T (2012) Epigenetic histone modification of Epstein-Barr virus BZLF1 promoter during latency and reactivation in Raji cells. J Virol 86:4752-4761. https://doi.org/10.1128/ JVI.06768-11

76. Murata T, Tsurumi T (2014) Switching of EBV cycles between latent and lytic states. Rev Med Virol 24:142-153. https://doi.org/ 10.1002/rmv. 1780

77. Muylaert I, Elias P (2010) Contributions of nucleotide excision repair, DNA polymerase eta, and homologous recombination to replication of UV-irradiated herpes simplex virus type 1. J Biol Chem 285:13761-13768. https://doi.org/10.1074/jbc.M110. 107920

78. Nagaraju T, Sugden AU, Sugden B (2019) Four-dimensional analyses show that replication compartments are clonal factories in which Epstein-Barr viral DNA amplification is coordinated. Proc Natl Acad Sci U S A 116:24630-24638. https://doi.org/10.1073/ pnas.1913992116

79. Nilsson T, Zetterberg H, Wang YC, Rymo L (2001) Promoterproximal regulatory elements involved in oriP-EBNA1independent and -dependent activation of the Epstein-Barr virus C promoter in B-lymphoid cell lines. J Virol 75:5796-5811. https://doi.org/10.1128/JVI.75.13.5796-5811.2001

80. Parelho V, Hadjur S, Spivakov M, Leleu M, Sauer S, Gregson HC, Jarmuz A, Canzonetta C, Webster Z, Nesterova T, Cobb BS, Yokomori K, Dillon N, Aragon L, Fisher AG, Merkenschlager M (2008) Cohesins functionally associate with CTCF on mammalian chromosome arms. Cell. 132:422-433. https://doi.org/10. 1016/j.cell.2008.01.011

81. Park R, Heston L, Shedd D, Delecluse HJ, Miller G (2008) Mutations of amino acids in the DNA-recognition domain of Epstein-Barr virus ZEBRA protein alter its sub-nuclear localization and affect formation of replication compartments. Virology. 382:145-162. https://doi.org/10.1016/j.virol.2008.09.009

82. Pentland I, Parish JL (2015) Targeting CTCF to control virus gene expression: a common theme amongst diverse DNA viruses. Viruses. 7:3574-3585. https://doi.org/10.3390/v7072791

83. Petosa C, Morand P, Baudin F, Moulin M, Artero JB, Muller CW (2006) Structural basis of lytic cycle activation by the Epstein-Barr virus ZEBRA protein. Mol Cell 21:565-572. https://doi.org/10. 1016/j.molcel.2006.01.006

84. Pfuller R, Hammerschmidt W (1996) Plasmid-like replicative intermediates of the Epstein-Barr virus lytic origin of DNA replication. J Virol 70:3423-3431

85. Pulvertaft JV (1964) Cytology of Burkitt's tumour (African lymphoma). Lancet. 1:238-240

86. Ragoczy T, Heston L, Miller G (1998) The Epstein-Barr virus Rta protein activates lytic cycle genes and can disrupt latency in B lymphocytes. J Virol 72:7978-7984

87. Ramasubramanyan S, Kanhere A, Osborn K, Flower K, Jenner RG, Sinclair AJ (2012a) Genome-wide analyses of Zta binding to the Epstein-Barr virus genome reveals interactions in both early and late lytic cycles and an epigenetic switch leading to an altered binding profile. J Virol 86:12494-12502. https://doi.org/10.1128/ JVI.01705-12

88. Ramasubramanyan S, Osborn K, Al-Mohammad R, Naranjo Perez-Fernandez IB, Zuo J, Balan N, Godfrey A, Patel H, Peters G, Rowe M, Jenner RG, Sinclair AJ (2015) Epstein-Barr virus transcription factor Zta acts through distal regulatory elements to directly control cellular gene expression. Nucleic Acids Res 43: 3563-3577. https://doi.org/10.1093/nar/gkv212

89. Ramasubramanyan S, Osborn K, Flower K, Sinclair AJ (2012b) Dynamic chromatin environment of key lytic cycle regulatory regions of the Epstein-Barr virus genome. J Virol 86:1809-1819. https://doi.org/10.1128/JVI.06334-11

90. Ray-Gallet D, Woolfe A, Vassias I, Pellentz C, Lacoste N, Puri A, Schultz DC, Pchelintsev NA, Adams PD, Jansen LE, Almouzni G (2011) Dynamics of histone H3 deposition in vivo reveal a nucleosome gap-filling mechanism for H3.3 to maintain chromatin integrity. Mol Cell 44:928-941. https://doi.org/10.1016/j.molcel. 2011.12.006

91. Rodriguez A, Jung EJ, Flemington EK (2001) Cell cycle analysis of Epstein-Barr virus-infected cells following treatment with lytic cycle-inducing agents. J Virol 75:4482-4489. https://doi.org/10. 1128/JVI.75.10.4482-4489.2001

92. Santos F, Peters AH, Otte AP, Reik W, Dean W (2005) Dynamic chromatin modifications characterise the first cell cycle in mouse embryos. Dev Biol 280:225-236. https://doi.org/10.1016/j.ydbio. 2005.01.025

93. Schaeffner M, Mrozek-Gorska P, Buschle A, Woellmer A, Tagawa T, Cernilogar FM, Schotta G, Krietenstein N, Lieleg C, Korber P, Hammerschmidt W (2019) BZLF1 interacts with chromatin remodelers promoting escape from latent infections with EBV. https://www.life-science-alliance.org. 2https://doi.org/10. 26508/lsa.201800108

94. Schepers A, Pich D, Hammerschmidt W (1993) A transcription factor with homology to the AP-1 family links RNA transcription and DNA replication in the lytic cycle of Epstein-Barr virus. EMBO J 12:3921-3929

95. Schepers A, Pich D, Hammerschmidt W (1996) Activation of oriLyt, the lytic origin of DNA replication of Epstein-Barr virus, by BZLF1. Virology. 220:367-376. https://doi.org/10.1006/viro.1996.0325

96. Schmid M, Speiseder T, Dobner T, Gonzalez RA (2014) DNA virus replication compartments. J Virol 88:1404-1420. https:// doi.org/10.1128/JVI.02046-13

97. Shannon-Lowe C, Rickinson A (2019) The global landscape of EBV-associated tumors. Front Oncol 9:713. https://doi.org/10. 3389/fonc. 2019.00713

98. Speck SH, Chatila T, Flemington E (1997) Reactivation of Epstein-Barr virus: regulation and function of the BZLF1 gene. Trends Microbiol 5:399-405. https://doi.org/10.1016/S0966842X(97)01129-3

99. Sugimoto A, Sato Y, Kanda T, Murata T, Narita Y, Kawashima D, Kimura H, Tsurumi T (2013) Different distributions of EpsteinBarr virus early and late gene transcripts within viral replication compartments. J Virol 87:6693-6699. https://doi.org/10.1128/ JVI.00219-13

100. Sugimoto A, Yamashita Y, Kanda T, Murata T, Tsurumi T (2019) Epstein-Barr virus genome packaging factors accumulate in BMRF1-cores within viral replication compartments. PLoS One 14:e0222519. https://doi.org/10.1371/journal.pone.0222519

101. Takacs M, Banati F, Koroknai A, Segesdi J, Salamon D, Wolf H, Niller HH, Minarovits J (2010) Epigenetic regulation of latent Epstein-Barr virus promoters. Biochim Biophys Acta 1799:228235. https://doi.org/10.1016/j.bbagrm.2009.10.005

102. Takada K, Shimizu N, Sakuma S, Ono Y (1986) Transactivation of the latent Epstein-Barr virus (EBV) genome after transfection of the EBV DNA fragment. J Virol 57:1016-1022 
103. Takada K, Horinouchi K, Ono Y, Aya T, Osato T, Takahashi M, Hayasaka S (1991) An Epstein-Barr virus-producer line Akata: establishment of the cell line and analysis of viral DNA. Virus Genes 5:147-156

104. Takagi S, Takada K, Sairenji T (1991) Formation of intranuclear replication compartments of Epstein-Barr virus with redistribution of BZLF1 and BMRF1 gene products. Virology. 185:309-315

105. Tempera I, Klichinsky M, Lieberman PM (2011) EBV latency types adopt alternative chromatin conformations. PLoS Pathog 7:e1002180. https://doi.org/10.1371/journal.ppat.1002180

106. Tempera I, Wiedmer A, Dheekollu J, Lieberman PM (2010) CTCF prevents the epigenetic drift of EBV latency promoter Qp. PLoS Pathog 6:e1001048. https://doi.org/10.1371/journal. ppat. 1001048

107. Thorley-Lawson DA, Gross A (2004) Persistence of the EpsteinBarr virus and the origins of associated lymphomas. N Engl J Med 350:1328-1337. https://doi.org/10.1056/NEJMra032015

108. Tsai K, Chan L, Gibeault R, Conn K, Dheekollu J, Domsic J, Marmorstein R, Schang LM, Lieberman PM (2014) Viral reprogramming of the Daxx histone H3.3 chaperone during early Epstein-Barr virus infection. J Virol 88:14350-14363. https://doi. org/10.1128/JVI.01895-14

109. Tsai K, Messick TE, Lieberman PM (2015) Disruption of host antiviral resistances by gammaherpesvirus tegument proteins with homology to the FGARAT purine biosynthesis enzyme. Curr Opin Virol 14:30-40. https://doi.org/10.1016/j.coviro.2015.07. 008

110. Tsai K, Thikmyanova N, Wojcechowskyj JA, Delecluse HJ, Lieberman PM (2011) EBV tegument protein BNRF1 disrupts DAXX-ATRX to activate viral early gene transcription. PLoS Pathog 7:e1002376. https://doi.org/10.1371/journal.ppat.1002376

111. Tsurumi T, Fujita M, Kudoh A (2005) Latent and lytic EpsteinBarr virus replication strategies. Rev Med Virol 15:3-15. https:// doi.org/10.1002/rmv.441

112. Uhlmann F (2008) Molecular biology: cohesin branches out. Nature. 451:777-778. https://doi.org/10.1038/451777a

113. Wang F, Petti L, Braun D, Seung S, Kieff E (1987) A bicistronic Epstein-Barr virus mRNA encodes two nuclear proteins in latently infected, growth-transformed lymphocytes. J Virol 61:945-954
114. Wen W, Iwakiri D, Yamamoto K, Maruo S, Kanda T, Takada K (2007) Epstein-Barr virus BZLF1 gene, a switch from latency to lytic infection, is expressed as an immediate-early gene after primary infection of B lymphocytes. J Virol 81:1037-1042. https:/ doi.org/10.1128/JVI.01416-06

115. Wendt KS, Yoshida K, Itoh T, Bando M, Koch B, Schirghuber E, Tsutsumi S, Nagae G, Ishihara K, Mishiro T, Yahata K, Imamoto F, Aburatani H, Nakao M, Imamoto N, Maeshima K, Shirahige K, Peters JM (2008) Cohesin mediates transcriptional insulation by CCCTC-binding factor. Nature. 451:796-801. https://doi.org/10. 1038/nature06634

116. Woellmer A, Arteaga-Salas JM, Hammerschmidt W (2012) BZLF1 governs CpG-methylated chromatin of Epstein-Barr virus reversing epigenetic repression. PLoS Pathog 8:e1002902. https:// doi.org/10.1371/journal.ppat.1002902

117. Woellmer A, Hammerschmidt W (2013) Epstein-Barr virus and host cell methylation: regulation of latency, replication and virus reactivation. Curr Opin Virol 3:260-265. https://doi.org/10.1016/j. coviro.2013.03.005

118. Yan J, Enge M, Whitington T, Dave K, Liu J, Sur I, Schmierer B, Jolma A, Kivioja T, Taipale M, Taipale J (2013) Transcription factor binding in human cells occurs in dense clusters formed around cohesin anchor sites. Cell. 154:801-813. https://doi.org/ 10.1016/j.cell.2013.07.034

119. Yu KP, Heston L, Park R, Ding Z, Wang'ondu R, Delecluse HJ, Miller G (2013) Latency of Epstein-Barr virus is disrupted by gain-of-function mutant cellular AP-1 proteins that preferentially bind methylated DNA. Proc Natl Acad Sci U S A 110:8176-8181. https://doi.org/10.1073/pnas.1301577110

120. Zaret KS, Mango SE (2016) Pioneer transcription factors, chromatin dynamics, and cell fate control. Curr Opin Genet Dev 37: 76-81. https://doi.org/10.1016/j.gde.2015.12.003

121. Zhang K, Lv DW, Li R (2017) B cell receptor activation and chemical induction trigger caspase-mediated cleavage of PIAS1 to facilitate Epstein-Barr virus reactivation. Cell Rep 21:34453457. https://doi.org/10.1016/j.celrep.2017.11.071

Publisher's note Springer Nature remains neutral with regard to jurisdictional claims in published maps and institutional affiliations. 